\title{
Noncrossing Monochromatic Subtrees and Staircases in 0-1 Matrices
}

\author{
Siyuan Cai, ${ }^{1}$ Gillian Grindstaff, ${ }^{2}$ András Gyárfás, ${ }^{3}$ and Warren Shull ${ }^{4}$ \\ ${ }^{1}$ Department of Mathematics, Northwestern University, 2033 Sheridan Road, Evanston, IL 60208-2730, USA \\ ${ }^{2}$ Department of Mathematics, Pomona College, 640 North College Avenue, Claremont, CA 91711, USA \\ ${ }^{3}$ Alfréd Rényi Institute of Mathematics, Hungarian Academy of Sciences, Budapest, P.O. Box 127, Budapest 1364, Hungary \\ ${ }^{4}$ Department of Mathematics and Computer Science, Emory University, Atlanta, GA 30322, USA
}

Correspondence should be addressed to András Gyárfás; gyarfas.andras@renyi.mta.hu

Received 16 September 2013; Accepted 12 November 2013; Published 23 January 2014

Academic Editor: Wai Chee Shiu

Copyright (c) 2014 Siyuan Cai et al. This is an open access article distributed under the Creative Commons Attribution License, which permits unrestricted use, distribution, and reproduction in any medium, provided the original work is properly cited.

\begin{abstract}
The following question is asked by the senior author (Gyárfás (2011)). What is the order of the largest monochromatic noncrossing subtree (caterpillar) that exists in every 2-coloring of the edges of a simple geometric $K_{n, n}$ ? We solve one particular problem asked by Gyárfás (2011): separate the Ramsey number of noncrossing trees from the Ramsey number of noncrossing double stars. We also reformulate the question as a Ramsey-type problem for 0-1 matrices and pose the following conjecture. Every $n \times n$ 0-1 matrix contains $n-1$ zeros or $n-1$ ones, forming a staircase: a sequence which goes right in rows and down in columns, possibly skipping elements, but not at turning points. We prove this conjecture in some special cases and put forward some related problems as well.
\end{abstract}

\section{Introduction}

A geometric graph (see [1]) is a graph whose vertices are in the plane in general position and whose edges are straightline segments joining the vertices. A subgraph of a geometric graph is noncrossing if no two edges have a common interior point. A geometric bipartite graph $G(n, n)$ is a geometric graph, whose $2 n$ vertices are in two disjoint $n$-element sets $A$ and $B$, and its edges are some segments $a b$ with $a \in$ $A$ and $b \in B$. The following representation, apparently studied first in [2], seems to be a more natural subclass of balanced geometric bipartite graphs $G(n, n)$ (in fact a standard way of drawing bipartite graphs). The partite sets of $G$ in $R^{2}$ are $A=\left\{a_{1}=(1,0), a_{2}=(2,0), \ldots, a_{n}=\right.$ $(n, 0)\}$ and $B=\left\{b_{1}=(1,1), b_{2}=(2,1), \ldots, b_{n}=\right.$ $(n, 1)\}$ and the edge $a_{i} b_{j}$ is the line segment joining $a_{i} \in$ $A$ and $b_{j} \in B$. This representation is called a simple $G(n, n)$.

Analogues of Turán and Ramsey theories have been considered for geometric graphs, see; [1,3-9] and its references. Our starting point is the following Ramsey-type problem for simple $G(n, n)$-s.
Problem 1 (see [5]). Find $f(n)$, the order of the largest monochromatic noncrossing subtree that exists in every 2 coloring of the edges of a simple geometric $K_{n, n}$. The lower bound is $4 n / 5$ and the upper bound is $n$ for even and $n+1$ for odd $n$.

Noncrossing subgraphs of simple $G(n, n)$-s can be easily characterized.

Proposition 2 (see [10]). Every connected component of a noncrossing subgraph of simple $G(n, n)$ is a caterpillar (a tree in which the vertices of degree larger than one form a path).

In fact, the lower bound $4 n / 5 \leq f(n)$ is proved in a stronger form.

Theorem 3 (see [5]). In every 2-coloring of the simple $K_{n, n}$ there is a noncrossing monochromatic double star with at least $4 n / 5$ vertices. This bound is asymptotically best possible.

Since Theorem 3 is asymptotically sharp, it was asked in [5] whether one can separate $f(n)$ and $4 n / 5$. Here, we answer this question positively, although with a small margin. 
Theorem 4. Consider $(4 / 5+\varepsilon) n \leq f(n)$, where $\varepsilon=1 / 880$.

We give a new construction showing that the upper bound in Problem 1 can be decreased by one when $n$ is odd.

Proposition 5. Consider $f(n) \leq n$ for $n \geq 2$.

Theorem 3 and Proposition 5 show that the noncrossing condition is important because it is easy to see that for odd $n$ there is a monochromatic tree of order $n+1$ in every 2-coloring of the edges of $K_{n, n}$. We suspect that the bound of Proposition 5 is best possible and risk the following conjecture.

Conjecture 6. For every $n \geq 2, f(n)=n$.

We also reformulate Conjecture 6 (and Problem 1) into a more attractive (and perhaps more inspiring) form. Let $A$ be an $n \times n$ 0-1 matrix. A 0 -staircase is a sequence of zeroes in $A$ which goes right in rows and down in columns, possibly skipping elements, but zero at each turning point. A 1-staircase is defined similarly on ones of A. A homogeneous staircase in $A$ is either a 0 - or a 1 -staircase. The length of a homogeneous staircase is the number of elements in it. Let $\operatorname{st}(A)$ be the maximum among the lengths of the homogeneous staircases of $A$. Finally, set

$$
s t(n)=\min \{s t(A): A \text { is an } n \times n \text { 0-1 matrix }\} .
$$

An example with $n=7$ is the matrix $A$ below where a 1 staircase of length 6 is shown:

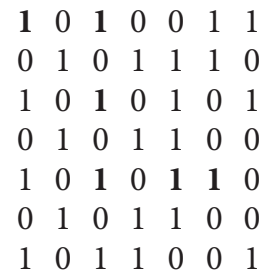

One can easily compute $s t(A)$ for any $n \times n$ matrix with the following procedure. We define an $n \times n$ matrix $B=$ $\left[b_{i, j}\right]$, the staircase matrix of $A$, where $b_{i, j}$ is the length of the longest homogeneous staircase of $A$ ending in position $(i, j)$. The matrix $B$ can be easily computed recursively from $A$, following a linear order of the elements of $B$, such that $b_{i, j}$ comes after all elements of $L(i, j)=\left\{b_{i, l}: 1 \leq l<j, a_{i, l}=a_{i, j}\right\}$ and also after all elements of $U(i, j)=\left\{b_{k, j}: 1 \leq k<i, a_{k, j}=\right.$ $\left.a_{i, j}\right\}$. Note that $L(i, 1)=U(1, j)=\emptyset$ for all $i$ and $j$. A natural linear order with this property can be defined by taking the first row left to right then the first column downwards starting at $b_{2,1}$ and repeating this for the remaining $(n-1) \times(n-1)$ matrix. Under this linear ordering, we define inductively

$$
b_{i, j}=1+\max \{\max L(i, j), \max U(i, j)\},
$$

where the maximum of the empty set is defined to be zero; for example,

$$
\begin{aligned}
b_{1,1} & =1+\max \{\max L(1,1), \max U(1,1)\} \\
& =1+\max \{\max \emptyset, \max \emptyset\}=1+\max \{0,0\}=1 .
\end{aligned}
$$

With the example matrix $A$, we get the following staircase matrix $B$, showing that $s t(A)=8$ and providing an easy way to trace back the 0 -staircase of length 8 in $A$ ending at $a_{6,7}$. The 0 -values are shown in bold:

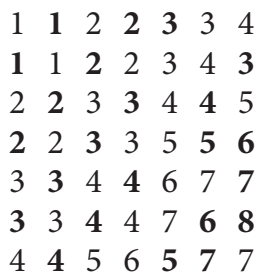

The following easy but important observation shows that the Ramsey-type problems for noncrossing subtrees in simple geometric $K_{n, n}$ and for staircases in $n \times n 0-1$ matrices are the same problems in different formulations.

Theorem 7. Consider $\operatorname{st}(n)=f(n)-1$.

Proof. A 2-coloring of the edges of a simple geometric $K_{n, n}$ can be considered as an $n \times n$ 0-1 matrix where the element in row $i$ and column $j$ is zero or one according to the color of the edge $a_{i} b_{j}$. By Proposition 2, every noncrossing monochromatic subtree is a caterpillar. The edges of the base path of the caterpillar correspond to turning points of a homogeneous staircase and the pending edges of the caterpillar correspond to elements in the same row or column between consecutive turning points (except at the beginning and the end). Thus, a monochromatic noncrossing subtree on $m$ vertices ( $m-1$ edges) corresponds to a homogeneous staircase of length $m-1$. The correspondence works backward as well, proving the theorem.

Theorem 7 allows to put forward Conjecture 6 in a more challenging form.

Conjecture 8. Every $n \times n$ 0-1 matrix has a homogeneous staircase of size $n-1$.

Another advantage of the staircase formulation is that it gives "a proof without words" to Proposition 5. Indeed, let $A$ be the $n \times n$ matrix with $a_{i, j}=1$ if $i+j \leq n$ or $i=j=n$ and zero otherwise (see below for $n=7$ ):

$$
\begin{array}{lllllll}
1 & 1 & 1 & 1 & 1 & 1 & 0 \\
1 & 1 & 1 & 1 & 1 & 0 & 0 \\
1 & 1 & 1 & 1 & 0 & 0 & 0 \\
1 & 1 & 1 & 0 & 0 & 0 & 0 \\
1 & 1 & 0 & 0 & 0 & 0 & 0 \\
1 & 0 & 0 & 0 & 0 & 0 & 0 \\
0 & 0 & 0 & 0 & 0 & 0 & 1
\end{array}
$$

One can immediately see that the longest homogeneous staircase of $A$ has $n-1$ elements, proving Proposition 5 . 
We have the following propositions supporting Conjecture 8 for various special matrices.

Proposition 9. Assume $a_{1,1}=a_{n, n}=0$ for the $n \times n$ matrix $A=\left[a_{i, j}\right]$ and there is no 0-staircase from $a_{1,1}$ to $a_{n, n}$. Then there is a 1-staircase of size at least $n-1$.

The next proposition settles Conjecture 8 for four of the 16 corner configurations of the lower left $2 \times 2$ submatrix of A.

Proposition 10. Suppose that the $n \times n$ matrix $A=\left[a_{i, j}\right]$ satisfies $a_{n, 1} \neq a_{n, 2}$ and $a_{n, 1} \neq a_{n-1,1}$. Then st $(A) \geq n-1$.

The next special case is when there are two consecutive columns whose binary sum is the all 1 vector (switching columns).

Proposition 11. Suppose that $A=\left[a_{i, j}\right]$ is an $n \times n$ 0-1 matrix with switching columns; that is, there exists $1 \leq j<n$ such that for every $1 \leq k \leq n$, one has $a_{k, j} \neq a_{k, j+1}$. Then $\operatorname{st}(A) \geq n-1$.

Finally, we prove Conjecture 8 for $n=8$ and note that its proof method still works for $n=9$ but would be much longer. On the other hand, the cases where $n<8$ are obvious and left to the reader.

Proposition 12. Every $8 \times 8$ 0-1 matrix has a monochromatic staircase of length at least 7 .

These propositions will be proved in Section 2, and Theorem 4 is proved in Section 3.

\section{Proofs of Special Cases of Conjecture 6}

Proof of Proposition 9. First, observe that $a_{1, n}=a_{n, 1}=1$, because otherwise there exists a 0 -staircase connecting $a_{1,1}$ to $a_{n, n}$. Suppose there are $a, b, c, d$ zeroes in the first row, first column, last row, and last column, respectively. A zero from the first row and a zero from the last row cannot share the same column, because otherwise a 0 -staircase connecting $a_{1,1}$ and $a_{n, n}$ would be present. Therefore, $a+c \leq n$; similarly $b+d \leq n$. Since $a_{1, n}=1$, the ones in the first row and in the last column form a 1-staircase of length $(n-a)+(n-$ d) $-1=2 n-a-d-1$. In the same way, there is a $1-$ staircase consisting of all ones in the first column and in the last row, of length $2 n-b-c-1$. The two lengths add up to $(2 n-a-d-1)+(2 n-b-c-1)=4 n-(a+c)-(b+d)-2 \geq 2 n-2$, so at least one of them must be at least $n-1$.

Proof of Proposition 10. Without loss of generality, assume $a_{n, 1}=0, a_{n-1,1}=a_{n, 2}=1$. Consider two cases as follows.

Case 1. $a_{n-1,2}=0$. Define $a$ to be the number of zeroes in column $1, b$ to be the number of zeroes in column $2, c$ to be the number of zeroes in row $n-1$, and $d$ to be the number of zeroes in row $n$. The following homogeneous staircases therefore exist:

(i) the 0 -staircase in column 1 and row $n$, turning at $a_{n, 1}$ with length $a+d-1$; (ii) the 0 -staircase in column 2 and row $n-1$, turning at $a_{n-1,2}$ with length $b+c-1$;

(iii) the 1-staircase in column 1 and row $n-1$, turning at $a_{n-1,1}$ with length $(n-a-2)+(n-c-2)+1$

(iv) the 1-staircase in column 2 and row $n$, turning at $a_{n, 2}$ with length $(n-b-2)+(n-d-2)+1$.

The sum of lengths of these four staircases is

$$
\begin{aligned}
a+ & d-1+b+c-1+(n-a-1)+(n-c-1)+1 \\
& +(n-b-1)+(n-d-1)+1=4 n-4
\end{aligned}
$$

so their average length is $n-1$; thus at least one of them must have length $n-1$ or more.

Case 2. $a_{n-1,2}=1$. Define $a$ to be the number of zeroes in column 1 and $b$ to be the number of zeroes in row $n$. The following homogeneous staircases therefore exist:

(i) the 0 -staircase in column 1 and row $n$, turning at $a_{n, 1}$ with length $a+b-1$;

(ii) The 1-staircase in column 1 and row $n, a_{n-1,2}$, turning at $a_{n-1,1}, a_{n-1,2}, a_{n, 2}$ with length $(n-a-2)+3(n-b-2)$.

The sum of lengths of these two staircases is $2 n-2$ so at least one of them must have length $n-1$ or more.

Proof of Proposition 11. Assume columns $j, j+1$ are switching columns. Let $r$ and $s$ be the smallest and largest values of $i$ for which $a_{i, j}=0$, and likewise let $p$ and $q$ are the smallest and largest values of $i$ for which $a_{i, j}=1$. We may assume $r, s, p$ and $q$ all exist and are different, otherwise, column $j$ contains at most one 1 or at most one 0 and the proof is finished.

Define $a$ to be the number of zeroes to the left of $a_{r, j}$ in row $r$, so there are $j-1-a$ ones there. Define $c$ to be the number of zeroes to the right of $a_{s, j+1}$ in row $s$, so there are $n-j-1-c$ ones there.

Define $d$ to be the number of ones to the left of $a_{p, j}$ in row $p$, so there are $j-1-d$ zeroes there. Define $e$ to be the number of ones to the right of $q, j+1$ in row $q$, so there are $n-j-1-e$ zeroes there.

Define $f$ to be the number of zeroes in column $j$, so there are $n-f$ ones in column $j$, and there are $n-f$ zeroes and $f$ ones in column $j+1$.

The 0 -staircase in row $r$, column $j$, and row $s$, which turns at $(r, j)$ and $(s, j)$, contains $a+f+c$ zeroes.

The 1 -staircase in row $r$, column $j+1$, and row $s$, which turns at $(r, j+1)$ and $(s, j+1)$, contains $(j-1-a)+f+(n-$ $j-1-c)$ ones.

The 1-staircase in row $p$, column $j$, and row $q$, which turns at $(p, j)$ and $(q, j)$, contains $d+(n-f)+e$ ones.

The 0 -staircase in row $p$, column $j+1$, and row $q$, which turns at $(p, j+1)$ and $(q, j+1)$, contains $(j-1-d)+(n-f)+$ $(n-j-1-e)$ zeroes.

The length sum of these homogeneous staircases is $a+f+$ $c+(j-1-a)+f+(n-j-1-c)+d+(n-f)+e+(j-1-$ $d)+(n-f)+(n-j-1-e)=4 n-4$, so the average length is $n-1$. Therefore, one of them has length at least $n-1$. 
Proof of Proposition 12. Let $A$ be an $8 \times 80$-1 matrix; without loss of generality, suppose $a_{8,8}=0$. By contradiction, assume all homogeneous staircases have length 6 or less. In the first row and last column combined, there can be at most 6 zeroes, so there must be at least 9 ones. Without loss of generality, assume the first row has at least as many ones as the last column, by assumption at most 6 .

Case 1. First row contains 6 ones. The column of the rightmost 1 must contain either 7 zeroes or a 1 , both giving a homogeneous staircase of length 7 , a contradiction.

Case 2. First row contains 5 ones; consequently, the last column contains at least 4 ones. Let $J$ be the set of indices $j$ with $a_{1, j}=1$ and let $I$ be the set of indices $i$ for which $a_{i, 8}=1$. We have $|I| \geq 4,|J|=5$. Then $a_{i, j}=0$, if $i$ is the smallest element of $I$ and $j$ is one of the last 4 elements of $J$ (otherwise we get a 1 -staircase of length at least 7). Similarly $a_{i, j}=0$, if $i$ is one of the first 4 elements of $I$ and $j$ is the largest element of $J$. This gives seven elements of $A$ with $a_{i, j}=0$, forming a 0 -staircase, a contradiction finishing the proof.

\section{Proof of Theorem 4}

Proof of Theorem 4. Consider an arbitrary red-blue coloring of the edges of a balanced geometric bipartite graph $G=$ $[A, B]$. Let $G_{R}$ and $G_{B}$ denote the red and blue subgraphs of G. Set

$$
D=\max \left\{d_{G_{R}}\left(a_{1}\right), d_{G_{R}}\left(b_{n}\right), d_{G_{B}}\left(a_{1}\right), d_{G_{B}}\left(b_{n}\right)\right\} .
$$

We will show that there is a noncrossing subtree in $G_{R}$ or in $G_{B}$ with $(4 / 5+\varepsilon) n$ vertices, where $\varepsilon$ is computed during the proof.

Assume first that $D \geq(6 / 10+2 \varepsilon) n$; without loss of generality, the maximum is attained at $b_{n}$ in the red color. Let $i$ denote the smallest index for which $a_{i} b_{n}$ is red. If $a_{i}$ has at least $(2 / 10-\varepsilon) n$ red neighbors in $B$, we have a red noncrossing double star on $a_{i} b_{n}$ spanning at least $(4 / 5+\varepsilon) n$ vertices. Otherwise $a_{i}$ has at least $(8 / 10+\varepsilon) n$ blue neighbors in $B$ giving a blue star that is as large as required.

From now on we consider the case $D<(6 / 10+$ $2 \varepsilon) n$; assume (w.l.o.g.) that edge $a_{1} b_{n}$ is red. Now-from the definition of $D$-both $d_{G_{R}}\left(a_{1}\right)$ and $d_{G_{R}}\left(b_{n}\right)$ are strictly greater than $(4 / 10-2 \varepsilon) n$; therefore, we have a noncrossing red double star $T$ on $a_{1} b_{n}$ with more than $(4 / 10-2 \varepsilon) n$ vertices in both $A$ and $B$. We may assume that $T$ has less than $(4 / 10+3 \varepsilon) n$ vertices on both $A$ and $B$; otherwise $T$ has at least $(8 / 10+\varepsilon) n$ vertices. Therefore, $a_{1}$ is adjacent in blue to a set $B_{1} \subset B$ such that $\left|B_{1}\right|>(6 / 10-3 \varepsilon) n$. Similarly, $b_{n}$ is adjacent in blue to a set $A_{1} \subset A$ such that $\left|A_{1}\right|>(6 / 10-3 \varepsilon) n$. Let $i$ be the smallest index for which $a_{i} \in A_{1}$ and $j$ is the largest index for which $b_{j} \in B_{1}$.

Observe that edges from $a_{i}$ to $B_{1}$ must be red apart from an initial segment $B_{2} \subset B_{1}$ such that $\left|B_{2}\right|<(2 / 10+4 \varepsilon) n$ because otherwise we have a noncrossing double star in blue of the required size. Similarly, edges from $b_{j}$ to $A_{1}$ must be red apart from an end segment of $A_{2} \subset A_{1}$ such that $\left|A_{2}\right|<$ $(2 / 10+4 \varepsilon) n$. Thus we have a red double star $T_{1}$ on the red edge $a_{i} b_{j}$ containing vertices of $A_{1}-A_{2} \cup B_{1}-B_{2}$. Thus $T_{1}$ has at least $(4 / 10-7 \varepsilon) n$ vertices in both $A$ and $B$ (and also at most $(4 / 10+8 \varepsilon) n$; otherwise we have the required large noncrossing red subtree). Note also that we may assume that $\left|A_{2}\right|,\left|B_{2}\right|>(2 / 10-11 \varepsilon) n$; otherwise if $\left|A_{2}\right| \leq(2 / 10-11 \varepsilon) n$, then

$$
\begin{aligned}
\left(\left|A_{1}-A_{2}\right|\right)+\left(\left|B_{1}-B_{2}\right|\right)> & \left(\left(\frac{6}{10}-3 \varepsilon\right)-\left(\frac{2}{10}-11 \varepsilon\right)\right) n \\
& +\left(\frac{4}{10}-7 \varepsilon\right) n=\left(\frac{8}{10}+\varepsilon\right) n .
\end{aligned}
$$

We note next that there are less than $(2 / 10+4 \varepsilon) n$ blue edges from $a_{i}$ to $B$; otherwise the blue star $A_{1} \cup\left\{b_{n}\right\}$ can be extended to a noncrossing double star on $(6 / 10-3 \varepsilon+2 / 10+$ $4 \varepsilon) n$ vertices. Thus more than $(1-(2 / 10+4 \varepsilon)) n=(8 / 10-4 \varepsilon) n$ red edges go from $a_{i}$ to $B$.

Set $S=\left\{b_{k}: j<k \leq n\right\}, R=\left\{b_{k}: 1 \leq k<j\right\}$ and observe that there are less than $(15 \varepsilon) n$ red edges from $a_{i}$ to $R-\left(B_{1}-B_{2}\right)$; otherwise we can extend $T_{1}$ to a noncrossing red double star with $(8 / 10-14 \varepsilon+15 \varepsilon) n=(8 / 10+\varepsilon) n$ vertices.

The two previous statements and $\left|B_{1}\right|-\left|B_{2}\right| \leq(4 / 10+8 \varepsilon) n$ imply that at least $((8 / 10-4 \varepsilon)-15 \varepsilon-(4 / 10+8 \varepsilon)) n=(4 / 10-$ $27 \varepsilon) n$ red edges go from $a_{i}$ to $S$. The same argument shows that at least $(4 / 10-27 \varepsilon) n$ red edges go from $b_{j}$ to the set $S_{1}=$ $\left\{a_{k}: 1 \leq k<a_{i}\right\}$. In particular, $|S|,\left|S_{1}\right| \geq(4 / 10-27 \varepsilon) n$.

Since $a_{i}$ sends at most $(15 \varepsilon) n$ red edges to $B_{2}$, it sends at least $\left(\left|B_{2}\right|-15 \varepsilon\right) n \geq(2 / 10-26 \varepsilon) n$ blue edges to set $B_{3} \subset B_{2}$. Suppose that the first vertex of $B_{3}\left(b_{t} \in B_{3}\right.$ with smallest $\left.t\right)$ sends $x$ blue edges to $S_{1}$. Then we have a blue noncrossing tree with at least

$$
\begin{aligned}
x+\left|B_{3}\right|+\left|A_{1}\right| & \geq x+\left(\frac{2}{10}-26 \varepsilon\right) n+\left(\frac{6}{10}-3 \varepsilon\right) n \\
& =x+\left(\frac{8}{10}-29 \varepsilon\right) n .
\end{aligned}
$$

Vertices; thus we may assume $x \leq(30 \varepsilon) n$. We conclude that some vertex of $B_{3}$ sends at least $\left(\left|S_{1}\right|-30 \varepsilon\right) n \geq(4 / 10-57 \varepsilon) n$ red edges to $U \subset S_{1}$.

Now, we define a noncrossing blue tree $T_{2}$ as follows. Let $b_{l}$ be the first element of $B_{1}-B_{2}$. Take the blue star from $a_{1}$ to $\left\{b_{p} \in B: p \leq l\right\}$; it has at least $\left|B_{2}\right| \geq(2 / 10-11 \varepsilon) n$ edges. Continue with the sequence of blue edges from $b_{l}$ to $U$ ending with the blue edge $b_{l} a_{k}, a_{k} \in U$. This sequence must contain at least $|U|-(15 \varepsilon) n \geq(4 / 10-72 \varepsilon) n$ vertices because at most $15 \varepsilon n$ red edges go from $b_{l}$ to $U$; otherwise $T_{1}$ would be extended. Finally, we add blue edges from $a_{k}$ to a subset of $\left\{b_{l}, b_{l+1}, \ldots, b_{n}\right\}$. To estimate how many, observe that there is a red star $T_{3}$ with center $a_{i}$ with at least $(4 / 10-7 \varepsilon) n$ leaves in $B_{1}-B_{2}$ and at least $(4 / 10-27 \varepsilon) n$ leaves in $S$, thus altogether at least $(8 / 10-34 \varepsilon) n$ leaves. If there is a red edge from $a_{k}$ to the $m$ th vertex of $T_{3}$ (from left), then we have a noncrossing red tree with at least $|U|+(8 / 10-34 \varepsilon) n-m \leq(8 / 10+\varepsilon) n$ vertices. 
This gives that $(4 / 10-57 \varepsilon) n+(8 / 10-34 \varepsilon) n-m \leq(8 / 10+\varepsilon) n$ and we conclude $(4 / 10-92 \varepsilon) n \leq m$. Altogether, $T_{2}$ has at least

$$
\begin{aligned}
\left(\frac{2}{10}\right. & -11 \varepsilon) n+\left(\frac{4}{10}-72 \varepsilon\right) n+\left(\frac{4}{10}-92 \varepsilon\right) n \\
& =(1-175 \varepsilon) n=\left(\frac{8}{10}+\varepsilon\right) n
\end{aligned}
$$

vertices when $\varepsilon=1 / 880$.

\section{Conflict of Interests}

The authors declare that there is no conflict of interests regarding the publication of this paper.

\section{Acknowledgment}

The authors are grateful for remarks of a referee, especially for pointing out that the following conjecture (stronger than Conjecture 6) is not true: every $n \times n 0-1$ matrix has a 0 staircase and a 1-staircase with total length at least $2 n-2$.

\section{References}

[1] J. Pach and P. K. Agarwal, Combinatorial Geometry, John Wiley \& Sons, New York, NY, USA, 1995.

[2] P. Eades and S. Whitesides, "Drawing graphs in two layers," Theoretical Computer Science, vol. 131, no. 2, pp. 361-374, 1994.

[3] N. Alon and P. Erdös, "Disjoint edges in geometric graphs," Discrete \& Computational Geometry, vol. 4, no. 4, pp. 287-290, 1989.

[4] P. Brass, G. Károlyi, and P. Valtr, "A Turán-type extremal theory of convex geometric graphs," in Discrete and Computational Geometry: Algorithms Combination, vol. 25, pp. 275-300, Springer, Berlin, Germany, 2003.

[5] A. Gyárfás, "Ramsey and Turán-type problems for non-crossing subgraphs of bipartite geometric graphs," Annales Universitatis Scientiarum Budapestinensis de Rolando Eötvös Nominatae, vol. 54, pp. 45-56, 2011.

[6] G. Károlyi, J. Pach, and G. Tóth, "Ramsey-type results for geometric graphs. I," Discrete \& Computational Geometry, vol. 18, no. 3, pp. 247-255, 1997.

[7] G. Károlyi, J. Pach, G. Tóth, and P. Valtr, "Ramsey-type results for geometric graphs. II," Discrete \& Computational Geometry, vol. 20, no. 3, pp. 375-388, 1998.

[8] Y. Kupitz, "On pairs of disjoint segments in convex position in the plane, Convexityand Graph theory (Jerusalem 1981)," Annals of Discrete Mathematics, vol. 20, pp. 203-208, 1984.

[9] A. Kaneko and M. Kano, "Discrete geometry on red and blue points in the plane-a survey," in Discrete and Computational Geometry, Algorithms Combininatorics, vol. 25, pp. 551-570, Springer, Berlin, Germany, 2003.

[10] F. Harary and A. Schwenk, "A new crossing number for bipartite graphs," Utilitas Mathematica, vol. 1, pp. 203-209, 1972. 


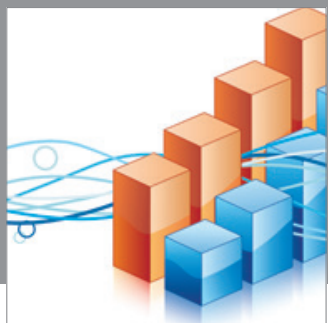

Advances in

Operations Research

mansans

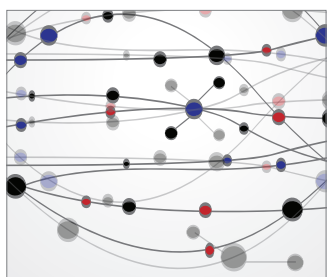

The Scientific World Journal
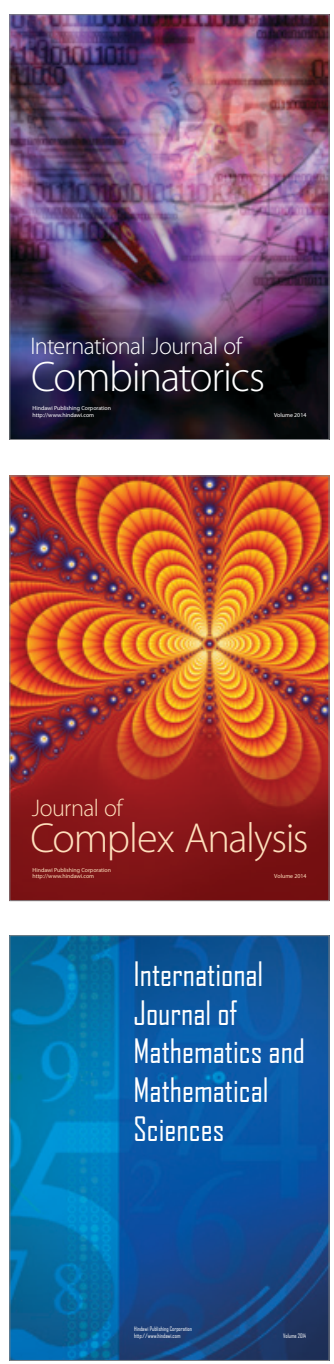
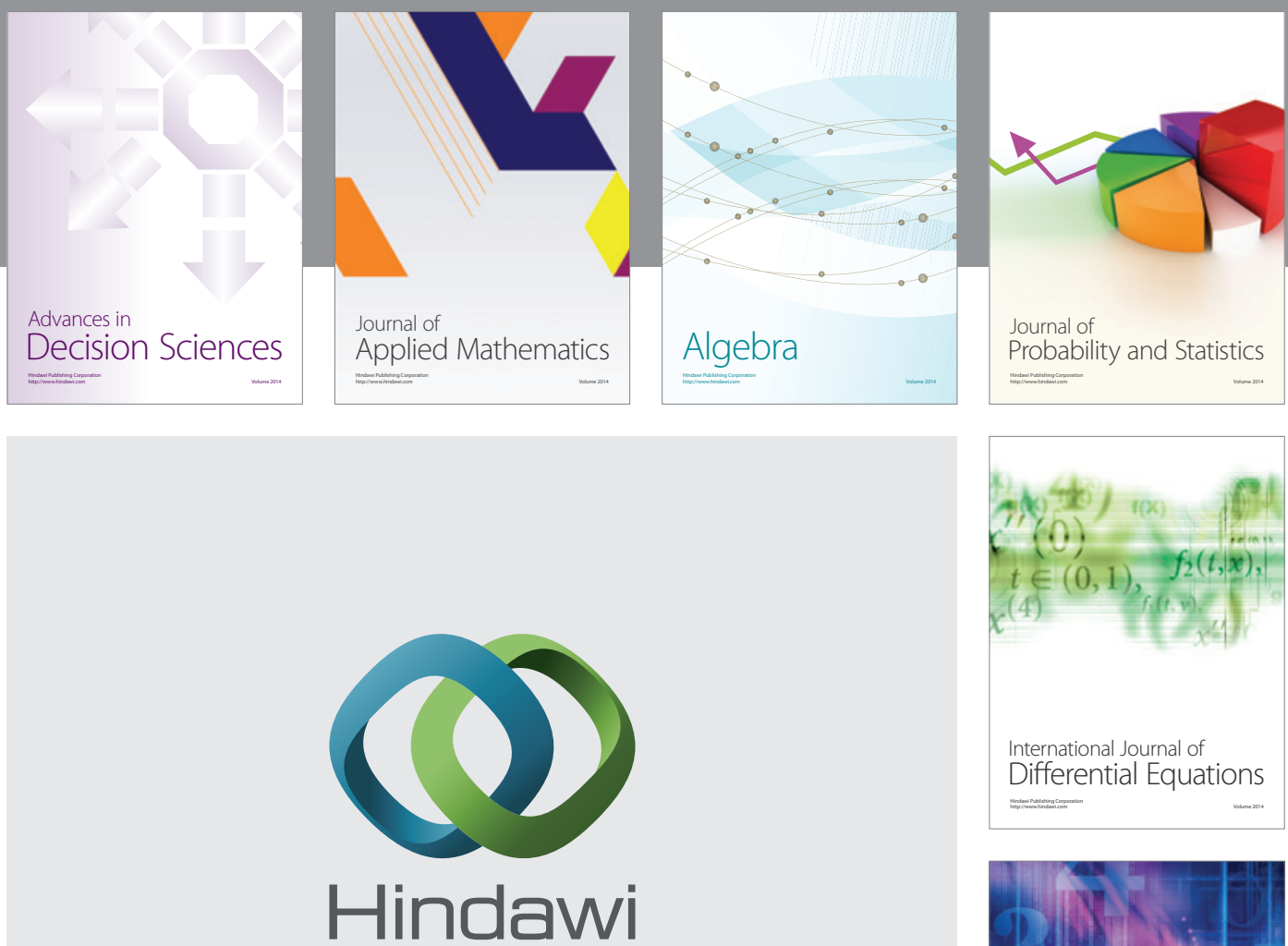

Submit your manuscripts at http://www.hindawi.com
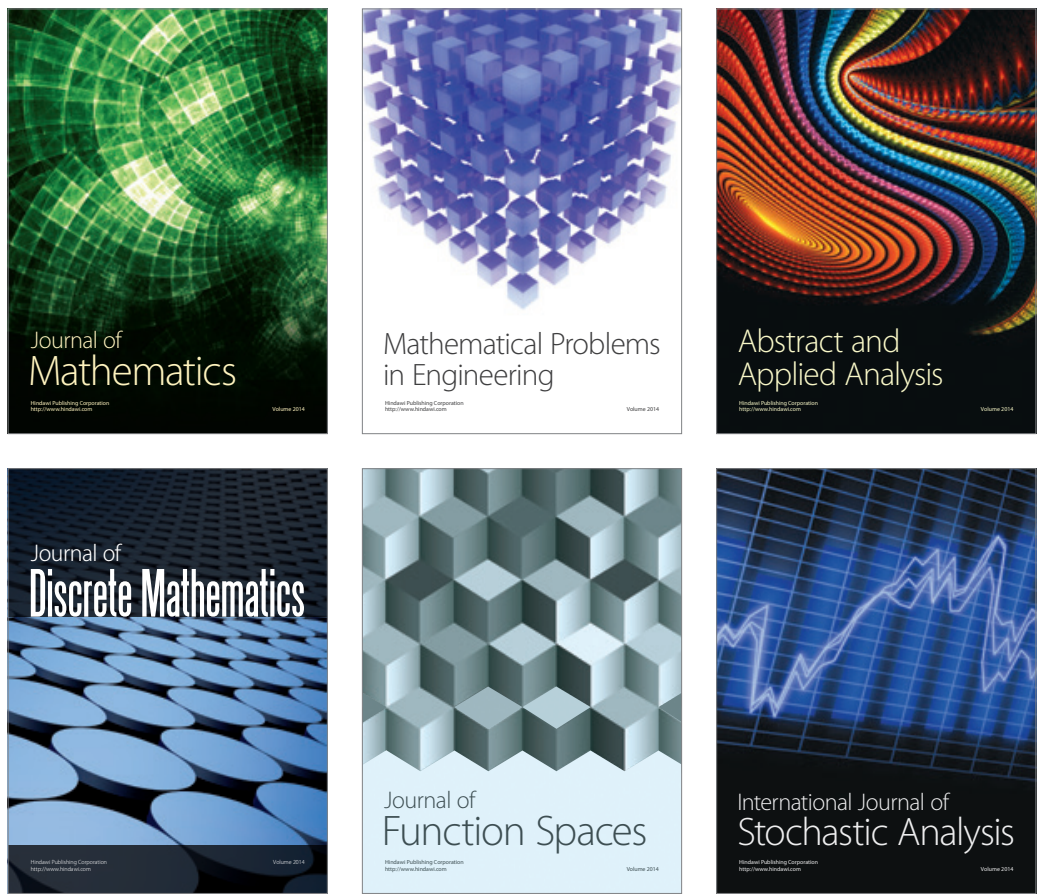

Journal of

Function Spaces

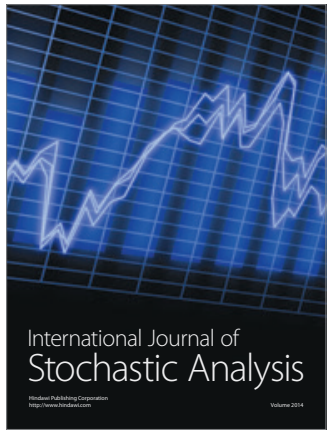

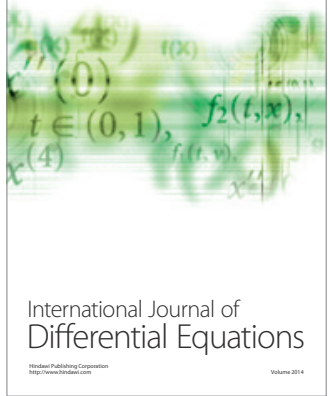
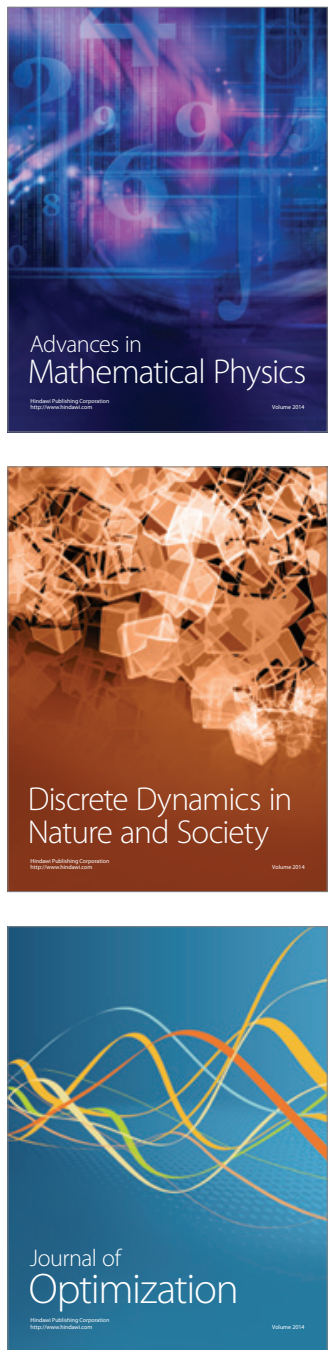